\title{
Can Aspergillus fumigatus conidia cause false-positive results in the galactomannan enzyme immunoassay test?
}

\author{
Ângela Leitzke Cabana ${ }^{[1]}$, Josiara Furtado Mendes ${ }^{[1]}$, Gabriel Baracy Klafke ${ }^{[2]}$, \\ Tchana Martinez Brandolt ${ }^{[3]}$, Aryse Martins Melo ${ }^{[4],[5]}$, Mário Carlos Araújo Meireles ${ }^{[1]}$ \\ and Melissa Orzechowski Xavier ${ }^{[2],[4]}$
}

\begin{abstract}
[1]. Programa de Pós-Graduação em Veterinária, Faculdade de Veterinária, Universidade Federal de Pelotas, Pelotas, RS, Brasil. [2]. Faculdade de Medicina, Universidade Federal do Rio Grande, Rio Grande, RS, Brasil.

[3]. Programa de Pós-Graduação em Ciências da Saúde, Faculdade de Medicina, Universidade Federal do Rio Grande, Rio Grande, RS, Brasil. [4]. Programa de Pós-Graduação em Parasitologia, Instituto de Biologia, Universidade Federal de Pelotas, Pelotas, RS, Brasil.

[5]. Centro de Recuperação de Animais Marinhos, Universidade Federal do Rio Grande, Rio Grande, RS, Brasil.
\end{abstract}

\begin{abstract}
Introduction: Several factors can cause false-positive results in the galactomannan (GM) test; however, others remain unknown. Presently, the impact of airborne contamination by Aspergillus conidia during enzyme-linked immunosorbent assay (ELISA) remains uninvestigated. Methods: We studied 12 A. fumigatus isolates. Fungal conidia were serially diluted and tested for GM detection using the Platelia ${ }^{\circledR}$ Aspergillus enzyme immunoassay (EIA). Results: The conidia concentration required for an EIApositive result was $4.8 \times 10^{3}$ (median). Conclusions: This is the first study to evaluate the impact of environmental contamination on the Platelia ${ }^{\circledR}$ Aspergillus EIA assay. Only massive contamination can interfere with GM optical readings, suggesting that environmental contamination does not cause false-positive test results.
\end{abstract}

Keywords: Invasive aspergillosis. Diagnosis. Platelia ${ }^{\circledR}$ Aspergillus EIA. Galactomannan. Air quality.

Galactomannan (GM) is a hydrosoluble polysaccharide derived from the fungal cell wall. Accordingly, GM detection plays an important role in the diagnosis of invasive fungal diseases, particularly invasive aspergillosis, since Aspergillus species produce large amounts of this antigen ${ }^{1,2}$. One of the main limitations of GM testing, however, is the frequent occurrence of false-positive results (up to $10 \%$ ), mostly due to the use of antibiotics from fungal origin, mucositis, and dialysis, in addition to cross-reaction with other fungal infections ${ }^{3-5}$.

Platelia ${ }^{\circledR}$ Aspergillus enzyme immunoassay (EIA) GM assay is highly sensitive (limit of detection in serum of $0.5 \mathrm{ng} /$ $\mathrm{mL})^{6,7}$. However, when considering that the air quality of indoor facilities, which may contain dozens to hundreds of Aspergillus conidia per square meter ${ }^{8}$, we wonder whether environmental contamination could be an additional source of false-positive results in the GM test. Thus, the aim of this study was to determine whether low-concentration contamination with Aspergillus fumigatus conidia could result in positive GM readings in the Platelia ${ }^{\circledR}$ Aspergillus EIA assay.

Corresponding author: Dra. Melissa Orzechowski Xavier e-mail: melissaxavierfurg@gmail.com

Received 9 August 2017

Accepted 17 November 2017
Twelve A. fumigatus isolates from the Mycology Laboratory of the Faculty of Medicine of the Federal University of Rio Grande (FURG, Brazil) were used in the study. Isolates were obtained from patients with invasive aspergillosis $(n=3)$ and Magellanic penguins $(\mathrm{n}=3)$. In addition, environmental isolates ( $\mathrm{n}=3)$ and reference Aspergillus strains were obtained (AF10, AF71 and AF13073, kindly provided by Prof. David W. Denning, National Aspergillosis Centre, UK).

To obtain young colonies, subcultures of the isolates were carried out in potato dextrose agar (PDA) at $25^{\circ} \mathrm{C}$ for 48 hours. Sterile saline solution $(0.85 \%)$ supplemented with $200 \mu \mathrm{L}$ of Tween 80 were added to the cultures, and a scraping of the surface of the colonies was carried out to obtain the solution of fungal propagules. After $30 \mathrm{~min}$, the suspension was filtered using a sterile double layer of gauze to retain the higher particles, ensuring that only conidia remained. Conidia suspensions were adjusted to $80-82 \%$ transmittance (absorbance of 0.09-0.11) by spectrophotometry (700S FEMTO®) at $530 \mathrm{~nm}$. Subsequently, a 1:50 dilution in sterile saline solution was performed according to the protocol described by the Clinical \& Laboratory Standard Institute (CLSI) ${ }^{9}$. The amount of conidia in each inoculum was determined by the Pour Plate technique, in which results were expressed in colony forming units $/ \mathrm{mL}(\mathrm{CFU} / \mathrm{mL})$.

Three serial dilutions (1:10) of the standardized inoculum were tested for GM using a commercial kit (Platelia ${ }^{\circledR}$ 
Aspergillus EIA, according to the manufacturer's instructions). Positive, negative, and cut-off controls were incorporated into each assay. GM results were expressed as optical densities (ODs), and samples were considered positive if the GM index was $>0.5$. All experiments were performed in duplicate. Data were compiled, and statistical analysis (descriptive analyses and Kruskal-Wallis) was performed using the SPSS ${ }^{\circledR} 20.0$ program.

Table 1 shows the inoculum standardization in solution, ranging from $1.6 \times 10^{6}$ to $6.7 \times 10^{7} \mathrm{CFU} / \mathrm{mL}$. Considering the volume of inoculum used in each well $(300 \mu \mathrm{L})$ for the test, these concentrations were calculated to determine the conidia amount in each tested well by multiplying by 0.3 and the corresponding dilution $\left(10^{-1}\right.$ to $\left.10^{-3}\right)$. Therefore, the median conidia concentration required to generate a positive result in the Platelia ${ }^{\circledR}$ Aspergillus EIA test was determined as $4.8 \times$ $10^{3}$, ranging from $4.8 \times 10^{2}$ to $2 \times 10^{6}$. The amount of conidia required for positive GM readings did not correlate with the origin of the isolates $(\mathrm{kw}=0.082)$, with medians of $3.6 \times 10^{3}$ (humans), $6 \times 10^{3}$ (penguins), $1.2 \times 10^{3}$ (environmental) and $5.4 \times 10^{4}$ (reference strains). GM indices for those isolates showed minimal conidia concentrations, ranging from 0.519 in an environmental strain (concentration $=1.2 \times 10^{3}$ ) to 3.57 in a penguin aspergillosis strain (concentration $=1.2 \times 10^{6}$ ) (Figure 1).

To our knowledge, this is the first study to experimentally determine the required amount of $A$. fumigatus conidia to produce a positive test result in a GM reaction. We demonstrated that at least 500 conidia $\left(4.8 \times 10^{2}\right.$ to $\left.2 \times 10^{6}\right)$ of A. fumigatus

TABLE 1: Results of the inoculum standardization following the CLSI protocol for 12 Aspergillus fumigatus isolates used in this study.

\begin{tabular}{llc}
\hline Strain & Origin & Inoculum (CFU/mL) \\
\hline AF13013 & Reference strain & $6.7 \times 10^{7}$ \\
AF71 & Reference strain & $1.8 \times 10^{7}$ \\
AF10 & Reference strain & $2.8 \times 10^{6}$ \\
M1270 & Human aspergillosis & $1.2 \times 10^{7}$ \\
M1437 & Human aspergillosis & $2.6 \times 10^{6}$ \\
M1834 & Human aspergillosis & $3.1 \times 10^{7}$ \\
C33 & Penguin aspergillosis & $1.1 \times 10^{7}$ \\
C90 & Penguin aspergillosis & $2.0 \times 10^{6}$ \\
C272 & Penguin aspergillosis & $4.1 \times 10^{7}$ \\
PL2 & Environmental isolate & $2.0 \times 10^{6}$ \\
PL3 & Environmental isolate & $9.0 \times 10^{6}$ \\
PL63 & Environmental isolate & $1.6 \times 10^{6}$ \\
\hline
\end{tabular}

CLSI: Clinical and Laboratory Standard Institute; CFU: colony forming unit.

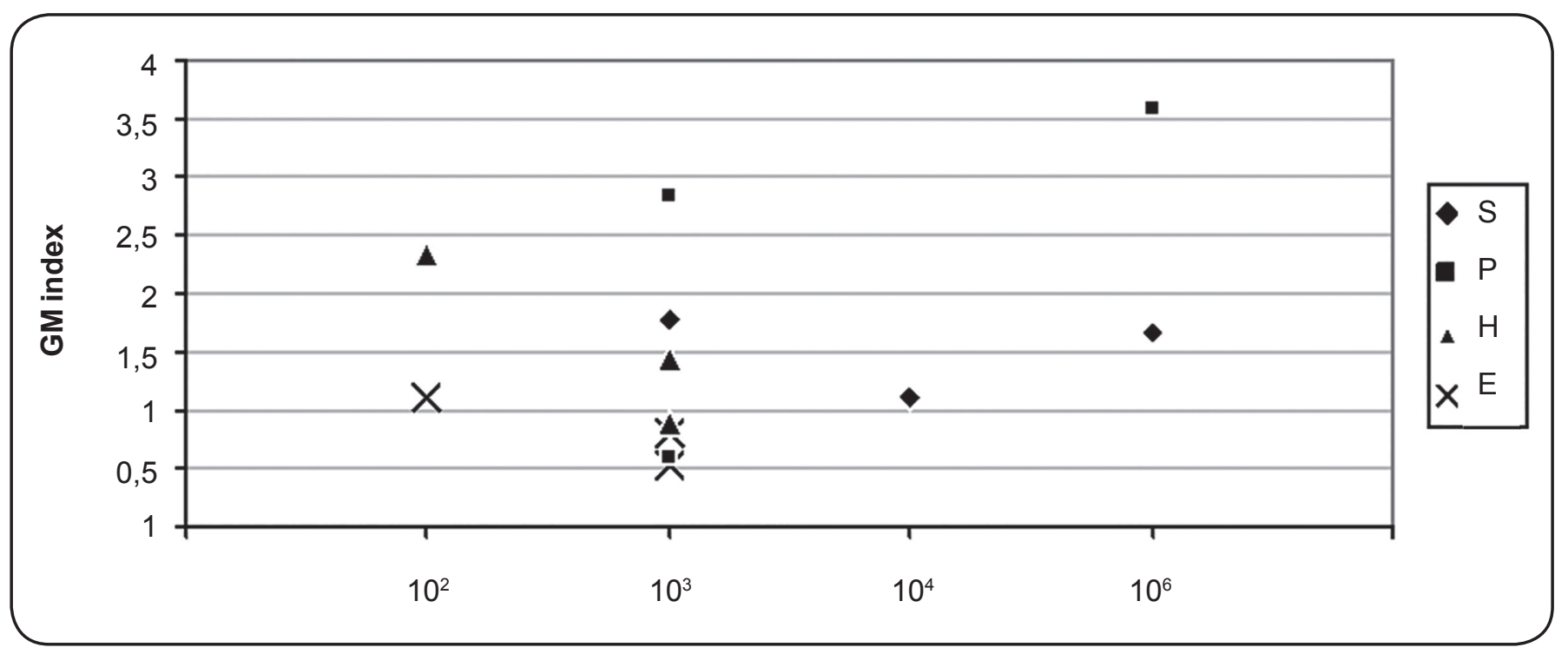

FIGURE 1: GM index at the lowest Aspergillus fumigatus conidial concentration, which causes positive results in the Platelia $\AA^{2}$ Aspergillus EIA $(n=12)$. S: standard strains; P: strains from penguin aspergillosis; H: strains from human aspergillosis; E: environmental strains; GM: galactomannan; EIA: enzyme immunoassay. 
are necessary to generate a positive result in the test (GM index above 0.5 ). Therefore, false-positive GM results due to this factor would require massive environmental contamination, which is not likely to occur in most clinical laboratories ${ }^{10}$.

We observed a wide variation in the amount of conidia required for a positive GM test result. Since Aspergillus hyphae release far more GM than Aspergillus conidia, strain-related differences in germination could explain these findings ${ }^{11,12}$. Differences in GM release have already been described between and within Aspergillus species, including in the same strains of A. fumigatus used in our study ${ }^{13,14}$.

Data found in our study contribute to the interpretation of Platelia ${ }^{\circledR}$ Aspergillus EIA results, demonstrating that the risk of a GM false-positive test result due to environmental contamination is low when performed following basic laboratory safety standards. Data of our study refer only to contamination by Aspergillus conidia, which is a limitation as other anemophily fungi (such as Penicillium and Fusarium) can also produce $\mathrm{GM}^{15}$ and were not tested in this context. Further studies are required to confirm or discharge the interference of other microorganisms in the Platelia ${ }^{\circledR}$ Aspergillus EIA.

\section{Conflict of interest}

The authors declare that there is no conflict of interest.

\section{REFERENCES}

1. Xavier MO, Aquino VR, Severo LC, Pasqualotto AC. Galactomanana no diagnóstico de aspergilose invasiva. Rev Bras Oncol Clínica. 2011;23(7):41-50.

2. Aquino VR, Goldani LZ, Pasqualotto AC. Update on the contribution of galactomannan for the diagnosis of invasive aspergillosis. Mycopathologia. 2007;163(4):191-202.

3. Xavier MO, Pasqualotto AC, Aquino VR, Sukiennik TCT, Severo LC. Galactomannan detection from piperacillin-tazobactam brands available in the Brazilian market. Braz J Infect Dis. 2009;13(5): 353-5.

4. Xavier MO, Pasqualotto AC, Cardoso ICE, Severo LC. Crossreactivity of Paracoccidioides brasiliensis, Histoplasma capsulatum, and Cryptococcus species in the commercial platelia
Aspergillus enzyme immunoassay. Clin Vaccine Immunol. 2009;16(1):132-3.

5. Monteiro AA, Rubenich DS, Zandoná MR, Pasqualotto AC. Impact of pre-analytical variables in the determination of serum galactomannan. Med Mycol. 2016;55(6):635-41.

6. Maertens JA, Klont R, Masson C, Theunissen K, Meersseman $\mathrm{W}$, Lagrou $\mathrm{K}$, et al. Optimization of the cutoff value for the Aspergillus double-sandwich enzyme immunoassay. Clin Infect Dis. 2007;44(10):1329-36.

7. Wheat LJ, Walsh TJ. Diagnosis of invasive aspergillosis by galactomannan antigenemia detection using an enzyme immunoassay. Eur J Clin Microbiol Infect Dis. 2008;27(4):245-51.

8. Sautour M, Sixt N, Dalle F, L'Ollivier C, Fourquenet V, Calinon, C, et al. Profiles and seasonal distribution of airborne fungi in indoor and outdoor environments at a French hospital. Sci Total Environ. 2009;407(12):3766-71.

9. Clinical and Laboratory Standards Institute (CLSI). Reference Method for Broth Dilution Antifungal Susceptibility Testing of Filamentous Fungi; Approved Standard. CLSI document M38-A2. $2^{\text {nd }}$ edition. Wayne: CLSI; 2008. p. 1-35.

10. Sautour M, Fournel I, Dalle F, Calinon C, L'Ollivier C, Goyer M, et al. Dynamics of fungal colonization in a new medical mycology laboratory. J Mycol Med. 2012;22(1):14-20.

11. Araujo R, Rodrigues AG. Variability of germinative potential among pathogenic species of Aspergillus. J Clin Microbiol. 2004;42(9):4335-7.

12. Mennink-kersten MASH, Ruegebrink D, Wasei N, Melchers WJG, Verweij PE. In vitro release by Aspergillus fumigatus of galactofuranose antigens, 1,3-beta-D-glucan, and DNA, surrogate markers used for diagnosis of invasive aspergillosis. J Clin Microbiol. 2006;44(5):1711-8.

13. Mennink-kersten MA, Reugbrink D, Klont R, Verweij PE. Release of galactofuranose antigens by different Aspergillus species. In: $45^{\text {th }}$ Interscience Conference Antimicrobial Agents Chemotherapy; 2005; Washington, USA: American Society for Microbiology; 2005. p. M-153.

14. Xavier MO, Araujo JSV, Aquino VR, Severo CB, Guazzelli LS, Severo LC, et al. Variability in galactomannan detection by platelia Aspergillus EIA ${ }^{\mathrm{TM}}$ according to the Aspergillus species. Rev Inst Med Trop. 2013;55(3):145-7.

15. Cummings JR, Jamison GR, Boudreaux JW, Howles MJ, Walsh TJ, Hayden RT. Cross-reactivity of non-Aspergillus fungal species in the Aspergillus galactomannan enzyme immunoassay. Diagn Microbiol Infect Dis. 2007;59(1):113-5. 